\title{
Detailed observations of the rippled surface of Antarctic blue-ice areas
}

\author{
Righard Bintanja, ${ }^{1}$ Carleen H. Reijmer, ${ }^{1}$ Suzanne J. M. H. Hulscher ${ }^{2}$ \\ ${ }^{1}$ Institute for Marine and Atmospheric Research, Utrecht University P.O. Box 80005, 3508 TA Utrecht, The Netherlands \\ ${ }^{2}$ Department of Civil Engineering, University of Twente, P.O. Box 217, 7500 AE Enschede, The Netherlands
}

\begin{abstract}
This paper presents detailed observations of the regularly rippled surface on an Antarctic blue-ice area near Svea, at five sites. The wavelength of the ripples was found to be $20-24 \mathrm{~cm}$, while the wave height (crest-trough) was $1-2 \mathrm{~cm}$. The ripple crests are generally oriented perpendicular to the direction of the strongest winds. Repeated measurements show that wave heights increase throughout the summer, with most ablation occurring in the wave troughs. This implies that traditional methods of measuring ablation (such as stakes when a rod on the ice surface is used to define a mean surface height) tend to underestimate total ablation because they sample only crests. One site exhibited significant migration of the surface ripples of about $2 \mathrm{~cm} \mathrm{month}^{-1}$ in the downwind direction, whereas three other sites showed no significant wave movement. The formation and the specific characteristics of the surface ripples are most likely caused by self-amplifying interaction mechanisms between the free ice surface and the overlying turbulent atmosphere, which necessarily involve spatial variations in sublimation. A simple model was used to quantify the interaction between the surface ripples, the airflow aloft and the sublimation rate. The model is able to predict wavelengths and migration rates that are in reasonable agreement with the observations.
\end{abstract}

\section{INTRODUGTION}

Blue-ice areas (BIAs) are relatively common features on the Antarctic continent (Bintanja, 1999). Even though their combined surface area is estimated to be only $1 \%$ of the total, most mountain ranges protruding through the ice seem to harbour BIAs. BIAs were named after their bluish appearance, which contrasts sharply with the surrounding white snowfields (Schytt, 1961). Antarctic BIAs are also locations of meteorite accumulations, as was first discovered in 1969 (Cassidy and others, 1992). In addition, the relatively old ice exposed at the surface of BIAs can be used for palaeoclimatic purposes (e.g. Grootes, 1990; Van Roijen and others, 1995).

BIAs represent Antarctica's ablation "islands", where net accumulation is zero (although temporary snow coverage may occasionally occur) and the surface loses mass to the atmosphere by sublimation. Hence, the net surface mass balance of BIAs is negative. Their formation and maintenance are caused by specific meteorological conditions induced by nearby (usually upwind) mountains, aided by the special characteristics of blue ice (e.g. low albedo, aerodynamic smoothness) (Bintanja and Van den Broeke, 1995; Van den Broeke and Bintanja, 1995; Bintanja, 2000b; Bintanja and Reijmer, 2001).

The surface of BIAs is composed of solid, bare ice. As a result, BIAs were readily identified as potential runways for airplanes, and nowadays aircraft regularly use BIAs. Mellor and Swithinbank (1989) carried out an inventory of BIAs for this purpose. They noticed that the surface of most BIAs was not flat. Instead, regular ripples or scallops (cups) were often observed, which were judged to be a favourable feature for a landing strip, since they provide grip. Many other studies involving Antarctic BIAs mention the presence of surface ripples, often as a mere curiosity. Figure 1 shows a typical BIA surface. It is generally composed of extensive stretches of uniform ripples, or, in some cases, cups. We estimate that $>95 \%$ of the surface area of the several BIAs that we visited during the fieldwork for this research displayed surface ripples.

Weller (1968) measured the dimensions of surface ripples on a BIA near Mawson, East Antarctica, and found typical wavelengths and wave heights of 5 and $2 \mathrm{~cm}$, respectively. Mellor and Swithinbank (1989) measured typical dimensions of blue-ice ripples and cups near Mount Howe. They found that the wavelength and height of the ripples were 20 and $10 \mathrm{~cm}$, respectively, whereas the diameter and depth of the cups were 15 and $4 \mathrm{~cm}$, respectively. Even though it seems that ripples and scallops are general features of Antarctic BIA surfaces, there is clearly some diversity in the actual observed shapes, dimensions and patterns. This diversity is likely to reflect differences in the mechanisms leading to the formation and growth of the various surface forms.

At first sight, there are superficial similarities between blue-ice ripples and patterns in sandy beds, such as ripples on a wet beach and submarine sand waves. Patterns in a sandy bed are formed due to free instabilities in the coupled system of an erodible bed and water-flow motion. Material is transported laterally, and horizontal variations in sediment transport result in local erosion and/or deposition. The governing mechanisms for the sand-ripples-sea-waves system are presented by Blondeaux (1990), and for sand-waves-tidalmotion by Hulscher (1996). In contrast, the only possible mechanism for blue-ice ripple formation is sublimation, which essentially constitutes an erosive mass flux (solid ice transforms to water vapour), occurring whenever there is 


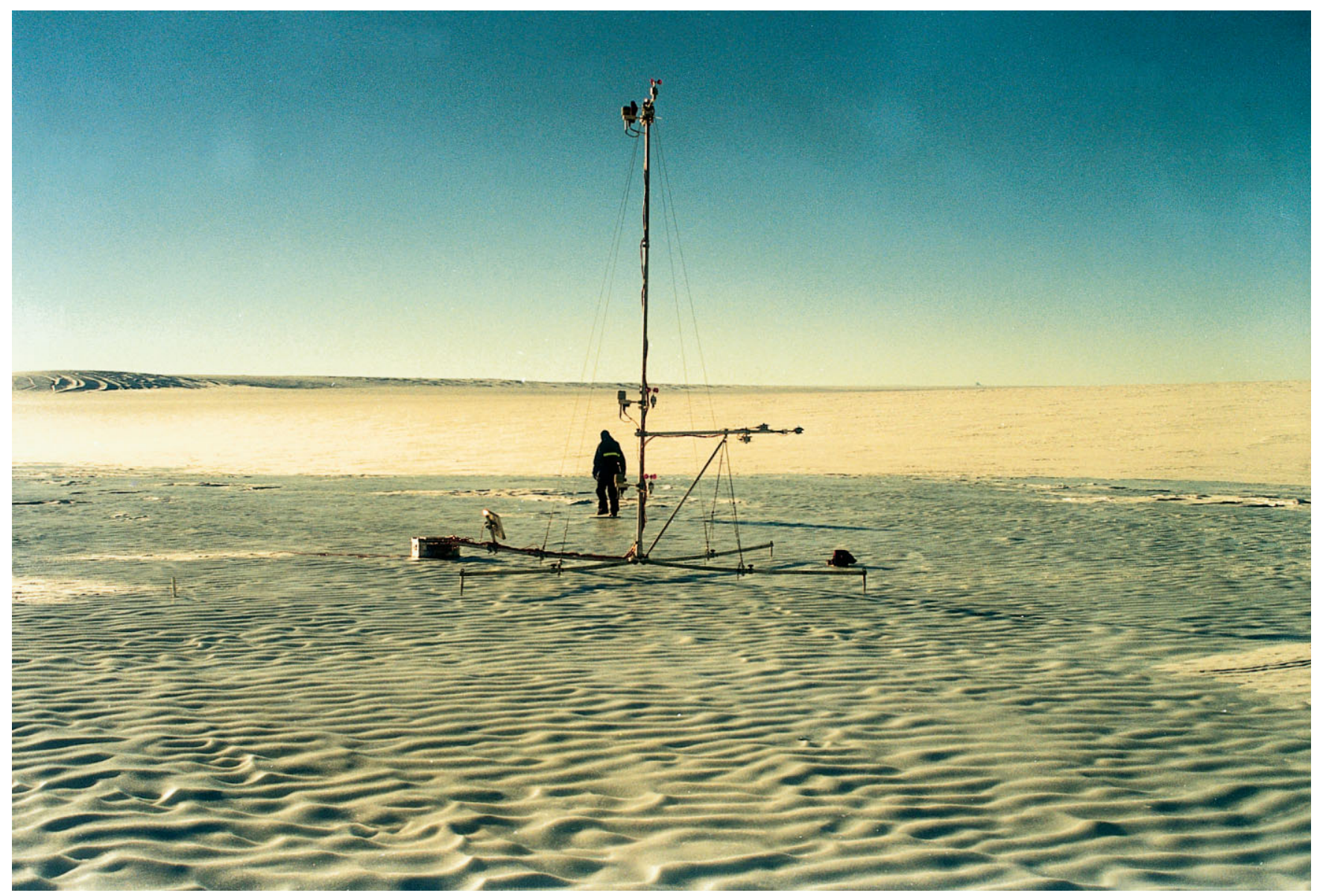

Fig. 1. Photograph of the surface of a BIA in the Heimefrontfjella, Dronning Maud Land, Antarctica. The foreground clearly showes a very regular ripple pattern. The height of the meteorological station is $6 \mathrm{~m}$.

wind forcing. Hence, rather than being formed by redistribution of sandy surface material, blue-ice ripples are somehow formed by regular spatial variations in sublimation rate. It should be stressed that, apart from the idea that both patterns arise due to free instabilities, these two ripple-producing mechanisms have little in common with regard to the physics involved. Other, less regular, surface forms commonly found on glaciers are caused mainly by differential melting induced by surface dust (Rhodes and others, 1987). One of the few exceptions to this are the quasi-regular penitents, sharp ice forms up to several meters high, which are typically found at low-latitude, high-altitude sites such as the South American Andes. Recently, micro-penitents have also been found in Antarctica (Winther and others, 1996). Their formation is related to differential sublimation under intense solar radiation (Lliboutry, 1954).

Thomas (1979) found that the characteristic dimensions of a scalloped and/or rippled morphology in flows over soluble or evaporative surfaces are determined primarily by the scale of the dominant eddies in the near-surface boundary layer. More specifically, he found that the wavelength $(\lambda)$ of the bedforms is linearly related to the inverse of the friction velocity $(u *$, which is proportional to the shear stress):

$$
\lambda=k \frac{\nu}{u *},
$$

where $\nu$ is the kinematic viscosity of the medium and $k$ is an empirical coefficient. By plotting $\lambda$ against $\nu / u *$ for a variety of bedforms with dimensions spanning more than four orders of magnitude (Fig. 2), he found a linear dependence with $k=10^{3}$. As can be seen in Figure 2, the observed dimen-

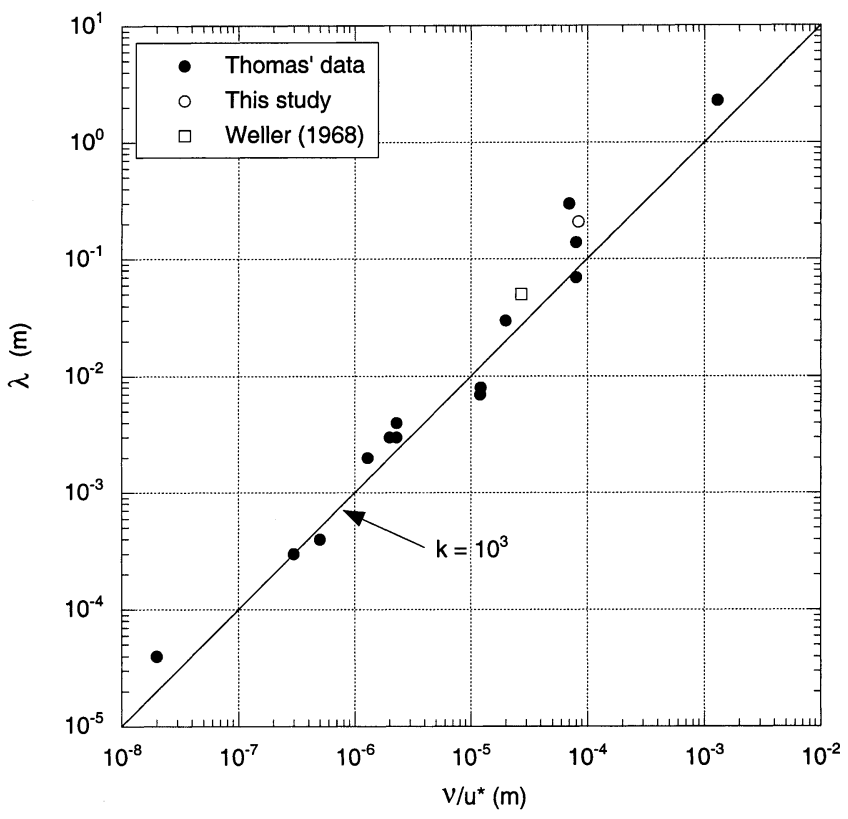

Fig. 2. Relationship between characteristic length scale of the bedforms (i.e. wavelength $\lambda$ ) and the ratio of kinematic viscosity and friction velocity for flows over soluble or evaporative walls. Data of Thomas (1979) pertain to flows of water or air over various types of material, such as aluminium, copper, bitumen, ice and plaster. The white circle and square indicate the blue-ice ripple data of this study and those given by Weller (1968), respectively. The solid line indicates the linear relation with $k=10^{3}$, as suggested by Thomas (1979). 


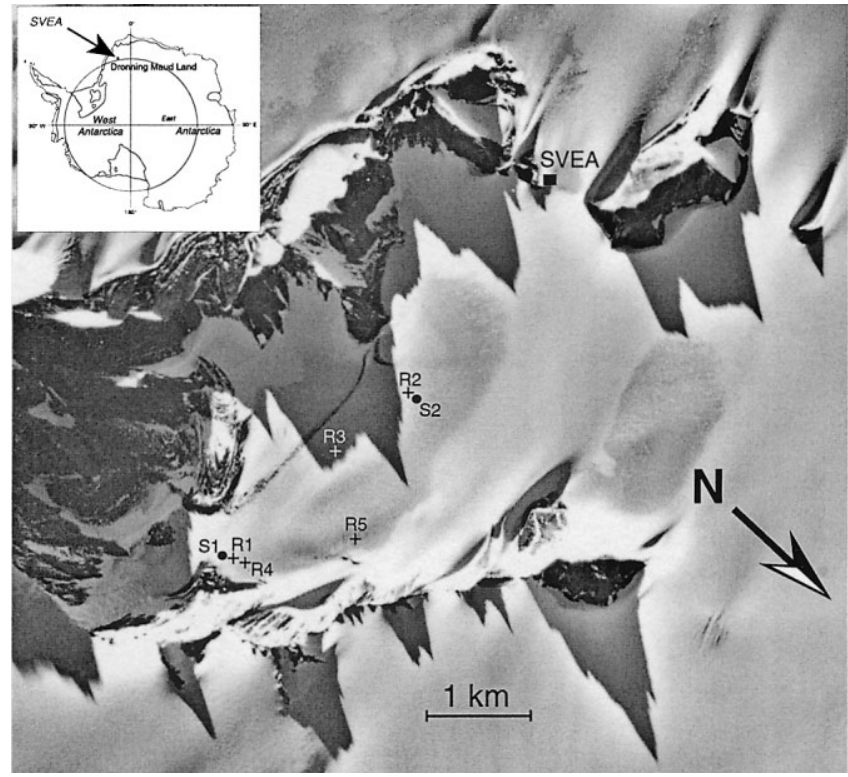

Fig. 3. Aerial photograph of the Scharffenbergbotnen valley in the Heimefrontfjella. Dark regions indicate bare rock and moraine, while light regions indicate snow-covered areas. Blueice areas are greyish in this picture. $R 1-R 5$ are the sites where ripple measurements have been carried out, while S1 and S2 indicate the positions of weather stations. Svea is in the western part of the valley. Meteorological data show that winds are generally from an easterly direction, as can also be deduced from the elongated snow dunes in this picture. The direction of the main ice flow is southeast into the valley. (Courtesy Institut für Angewandte Geodäsie, Frankfurt.)

sions of blue-ice ripples agree favourably with this empirical relationship (see also Bintanja, 1999). This indicates that blueice ripples may be a manifestation of a general mechanism producing ripples in flows over evaporative walls. This would then imply that these ripples represent imprints of predominant eddy patterns of the near-surface flow over blue ice.

At present, detailed observations of blue-ice ripples have not been made, nor has a satisfactory quantitative explanation for their existence been given. In order to begin filling these gaps, we will describe results of detailed measurements of the characteristics of blue-ice ripples, such as wavelength, wave height, wave speed and orientation with respect to the dominant wind direction. (Incidentally, in this paper, we will loosely use the terms "ripples" and "waves" to mean the same thing.) We will compare ablation rates measured traditionally by stakes with those obtained through detailed ripple observations. A simple model will be formulated in a first attempt to identify the mechanisms responsible for ripple formation.

\section{LOGATION AND OBSERVATIONS}

Blue-ice ripple measurements were carried out on a BIA near the Swedish research station Svea, which is located in the Heimefrontfjella, Dronning Maud Land, in austral summer 1997/98. The observations were made in the framework of a comprehensive and detailed meteorological programme carried out to gain knowledge of the meteorological and glaciological conditions of Antarctic BIAs (Bintanja and others, 1998). The meteorological experiment consisted of seven profile stations (three over blue ice and four over snow), at which wind speed, temperature and humidity, measured at

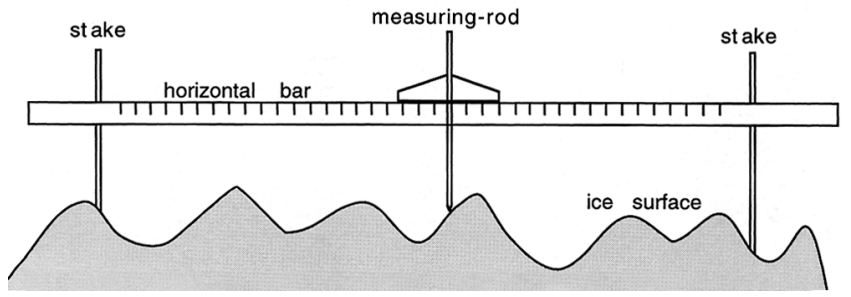

Fig. 4. Schematic illustration of the technique used to measure blue-ice ripple profiles. The two stakes were drilled into the ice. A bar with cm scale was connected horizontally to these stakes at fixed heights. A measuring device sliding manually along the bar was used to measure the vertical distance to the ice surface at fixed horizontal positions. The two stakes were also used to measure ablation in the conventional way.

three or five heights, as well as wind direction, the radiative fluxes and subsurface temperatures were recorded continuously during 37 days. Detailed turbulence measurements (with fast-response sensors), snowdrift measurements, ablation (stake method), cable-balloon and radiosonde soundings and synoptic observations were carried out at two sites. Bintanja (2000a) used this dataset to identify forcing mechanisms of large-scale flow, while Bintanja (2000c) evaluated and interpreted the surface energy balance at the sites. The 1997/98 experiment followed up a similar but less extensive field campaign in 1992/93 (Bintanja and Van den Broeke, 1995).

Figure 3 portrays the surroundings of Svea with emphasis on the main BIA in the Scharffenbergbotnen valley. It shows the five sites (R1-R5) where surface ripples were measured in detail, as well as the locations of two of the profile weather stations ( $\mathrm{S} 1$ and $\mathrm{S} 2$ ). Sites $\mathrm{R} 1$ and $\mathrm{R} 2$ were chosen close to $\mathrm{S} 1$ and $\mathrm{S} 2$ to potentially relate the observed ripple characteristics to nearby meteorological data, and to facilitate interpretation in a broader meteorological context. In general, the ripple sites were selected on the basis of the requirement that a stretch of at least $2 \mathrm{~m}$ of more-or-less uniform ripples should be present.

After a suitable location was selected, the procedure to measure surface profiles at each of the five sites was as follows. Two stakes were drilled vertically into the ice, about $2 \mathrm{~m}$ apart. Between them, a bar was attached exactly horizontally at about $10 \mathrm{~cm}$ above the mean surface level (see Fig. 4), in a direction perpendicular to the crests of the surface ripples. The exact height of the bar was marked on the two stakes, as the bar was removed after each profile measurement for use at the other sites. A small device, basically a high-precision measuring rod constructed to fit and slide over the horizontal bar (which contained a cm scale), was used to measure the vertical distance to the ice surface, with a precision of $0.5 \mathrm{~mm}$. Such a measurement was made every $2 \mathrm{~cm}$ to create a horizontal profile of ice height, consisting of 101 points over a $2 \mathrm{~m}$ stretch, which took about $1 \mathrm{~h}$. We will refer to this as the "profile measurement". Measurements were carried out at least twice at each site: at the beginning of the measuring period (29-30 December 1997; visit I) and at the end (2-4 February 1998; visit III). Measurements were also carried out at R1 and R2 in between (18 January 1998; visit II). Consequently, not only the specific forms and dimensions of blue-ice ripples were measured but also their possible changes in time. The summer is well suited for this because of maximum sublimation rates (Bintanja and others, 1997; Bintanja, 2000c). Therefore, any temporal changes in ripple characteristics are expected to be highest during summer. 
Surface ablation measurements over bare-ice surfaces are traditionally carried out using stakes. The ablation or accumulation rate is recorded by measuring temporal changes in the vertical distance between a rod placed on the ice surface and a marker on a stake drilled into the ice. The use of a rod in this way ensures that part of the surface with the length of the rod surrounding the stake is taken into account, and not just a point adjacent to the stake (at which the ablation is often influenced by the presence of the stake). Over blue ice and other irregular surfaces, this procedure implies that one measures the ablation of the highest surface elements, i.e. the ripple crests over blue ice. In this paper, the ablation as determined by the stake-ablation method will be compared with the average ablation along the $2 \mathrm{~m}$ profile to infer the accuracy of the usual stake measurements over blue ice. An automatic weather station (AWS) near site 1 was equipped with an acoustic height sensor, which continuously measured ice ablation. The accuracy of this sensor is estimated at $0.5 \mathrm{~cm}$, but depends on knowledge of the air temperature to correct for variations in the speed of sound. These sensor data will also be used to compare rippleablation results. At each site, the orientation of the surface ripples was determined at each visit.

Unfortunately, an exceptionally warm period at the beginning of January caused extensive surface melt (no subsurface melt features were detected). The surface ripples at R 3 and small portions of the profiles at R4 and R5 were destroyed. Since surface melt is presumably unrelated to the formation process of the ripples, temporal changes in ripple dimensions could not be determined in these cases. The surface meltwater gathered in two small lakes, which froze during the subsequent colder period. At the end of the measuring period, we noticed surface ripples on these lakes and hence witnessed their actual formation. The ripples on one of these lakes, close to R1, had a wavelength of $17 \mathrm{~cm}$ and a wave height of $1 \mathrm{~cm}$, and had formed within 1 month.

\section{RESULTS OF OBSERVATIONS}

\subsection{Ripple waveform}

Figure 5 shows the shape of the blue-ice surface at each of the five sites on visit I. The dominant wind direction (also that of the strongest winds) is from the right to the left side of the plot. At each site, the surface height varies in a regular manner. Obvious similarities between the five sites are the wavelength of about $20 \mathrm{~cm}$ and the wave height of about $1-2 \mathrm{~cm} . \mathrm{R} 2$ and especially R5 exhibit very regular and symmetric waves. In contrast, the profile at R1 especially, but also at R3 and R4, shows ripples of far more asymmetrical form. In fact, the ripples at $\mathrm{R} 1$ (and, to a lesser extent, at $\mathrm{R} 3$ and $\mathrm{R} 4$ ) exhibit a sawtooth pattern with the steepest part of the wave at the lee side. The surface forms at Rl and R4 actually resembled cups rather than ripples. According to Bintanja and Reijmer (2001), turbulence is most vigorous at $\mathrm{R} 1$ and $\mathrm{R} 4$ due to the proximity of the upwind valley wall (see Fig. 3). Therefore, it may well be that asymmetrically formed cups are more common in strong turbulent environments, while more uniform ripples tend to occur in places where turbulence is less intense.

\subsection{Ripple wavelength}

The average wavelength of the blue-ice ripples was measured in two ways. In the first method, we measured the total

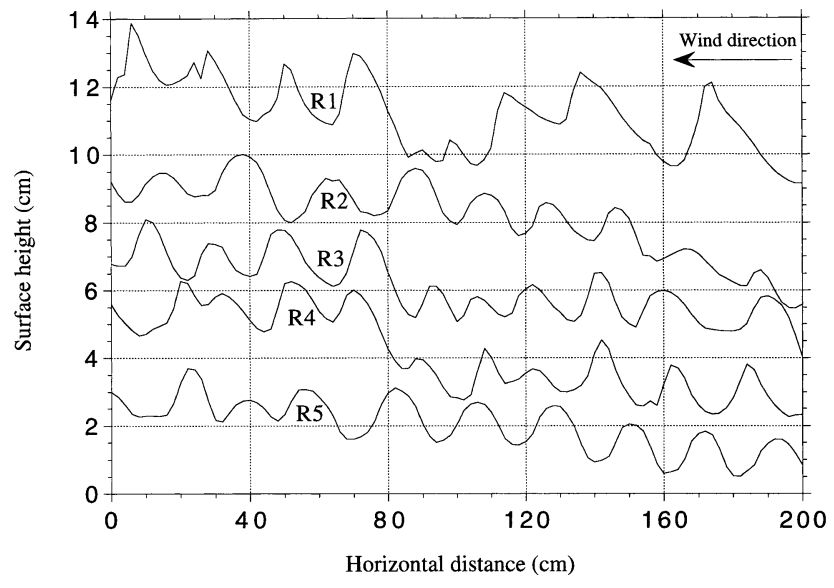

Fig. 5. Measured blue-ice profiles at each of the five sites on visit I (29-30 December 1997). Profiles are shifted vertically to facilitate intercomparison. Predominant winds are from right to left.

distance between the first and last crests of each profile shown in Figure 5 and divided that by the number of waves (profile method). In the second method, we visually selected 5-10 uniform ripple profiles whose average wavelength was determined over a stretch containing 5-10 wavelengths (selection method). The latter method was applied only at the first visit, since we did not expect significant temporal changes in ripple wavelength. The repeated profile measurements confirmed this.

Table 1 depicts the wavelengths determined by both methods at each site. It is clear that the ripple wavelengths at the Scharffenbergbotnen BIA range from 20 to $24 \mathrm{~cm}$, with no significant differences between the sites. Moreover, the results of both methods agree well at most sites. The values also agree well with the ripple wavelengths reported by Mellor and Swithinbank (1989), but are much larger than those observed by Weller (1968) who found a typical wavelength of $5 \mathrm{~cm}$. This is most likely due to differences in nearsurface meteorological conditions, in particular friction velocity (see Fig. 2). According to Equation (1), stronger winds and higher $u *$ values will lead to surface ripples with shorter wavelengths. During our frequent visits to this and other nearby BIAs we never encountered ripples with a markedly different wavelength.

\subsection{Ripple wave height}

Wave height was also measured in two ways. First, we determined successive crest-trough and trough-crest height differences from the observed profiles ("profile wave-height method"). Secondly, a horizontal rod was placed on top of two successive crests, and the vertical distance with the

Table 1. Average wavelength of the observed ripples (cm) at each site, determined by the "profile" and "selection" methods (see text for explanation)

\begin{tabular}{ccc}
\hline Site & Profile & Selection \\
& & \\
\hline R1 & 24.0 & $22.9 \pm 1.8$ \\
R2 & 21.1 & $20.9 \pm 2.0$ \\
R3 & 20.0 & $21.1 \pm 1.5$ \\
R4 & 20.5 & $21.5 \pm 2.2$ \\
R5 & 21.3 & $23.1 \pm 0.9$ \\
\hline
\end{tabular}

Note: The error denotes the standard deviation of the mean value. 
Table 2. Average ripple wave height (cm) (trough-crest) at each site, determined by the profile and bar methods (see text for explanation)

\begin{tabular}{|c|c|c|c|c|}
\hline \multirow[b]{2}{*}{ Site } & \multicolumn{2}{|c|}{$I$} & \multirow{2}{*}{$\begin{array}{c}I I \\
\text { Profile }\end{array}$} & \multirow{2}{*}{$\begin{array}{c}\text { III } \\
\text { Profile }\end{array}$} \\
\hline & Bar & Profile & & \\
\hline R1 & $1.94 \pm 0.38$ & $1.97 \pm 0.62$ & $2.06 \pm 0.86$ & $2.24 \pm 0.95$ \\
\hline R2 & $1.47 \pm 0.28$ & $1.10 \pm 0.37$ & $1.20 \pm 0.36$ & $1.46 \pm 0.51$ \\
\hline R3 & $1.60 \pm 0.32$ & $1.25 \pm 0.43$ & - & - \\
\hline R4 & $1.80 \pm 0.37$ & $1.25 \pm 0.50$ & - & $1.61 \pm 0.60$ \\
\hline R5 & $1.66 \pm 0.33$ & $1.26 \pm 0.32$ & - & $2.00 \pm 0.51$ \\
\hline
\end{tabular}

Notes: The profile method was used on each visit to each site (except at R3), while the bar method was only used on visit I. The error denotes the standard deviation of the mean value.

trough in between was measured ("bar method"). At each site, 10-20 bar measurements were carried out at "random" locations but, again, only at the first visit. Table 2 shows the results. The bar method appears to have yielded too large wave heights compared to the profile method at four of the five sites, although the differences are not statistically significant. Apparently, the visual selection procedure had resulted in a bias towards more prominent (and hence higher) waves, making our selection of waves in the second method not random at all. We should therefore disregard the results of the bar method. The profile method yields wave heights of $1.1-1.3 \mathrm{~cm}$ at R2-R5, and of almost $2 \mathrm{~cm}$ at R1. These values are much lower than the ripple heights of $10 \mathrm{~cm}$ reported by Mellor and Swithinbank (1989). They are also lower than what we loosely estimated from visual inspection before the actual measurements were performed. This raises the question whether the ripple heights reported by Mellor and Swithinbank (1989) were perhaps also overestimates, based on visual estimates only. Alternatively, meteorological conditions over the BIAs in the Heimefrontfjella region may favour ripples with relatively small amplitudes. The exact reasons for the presumed differences in wave height are currently unknown.

\subsection{Ripple evolution}

Wave height was found to increase with time at all sites (Table 2). During the 35 day period, average wave height increased between $0.27 \mathrm{~cm} \mathrm{(Rl)} \mathrm{and} 0.74 \mathrm{~cm}(\mathrm{R} 5)$. These values represent increases of $14-60 \%$, which strongly indicates that mechanisms favouring an increase in wave height were active during the measuring period. Moreover, the standard deviation also increases in time, which indicates an increase of the height differences between the waves. Apparently, the increase in wave height is not uniform. Generally, the ripples with the largest amplitude appear to grow more than the smaller ones. The consistency among the four sites indicates that the increase in wave height of blue-ice ripples may well be a general feature in summer. Interesting in this respect is the aforementioned observation of the formation of ripples with a wave height of $1 \mathrm{~cm}$ from an initially flat ice surface within 1 month. It is clear that ripple-forming mechanisms are active during summer when sublimation rates are at their peak.

\subsection{Ripple ablation}

The observed increase in wave height indicates that more ablation takes place in the wave troughs than at the crests. This is further illustrated in Figure 6, which shows the two measured profiles at R 5 as well as the difference between
Surface profiles at $\mathbf{R} 5$

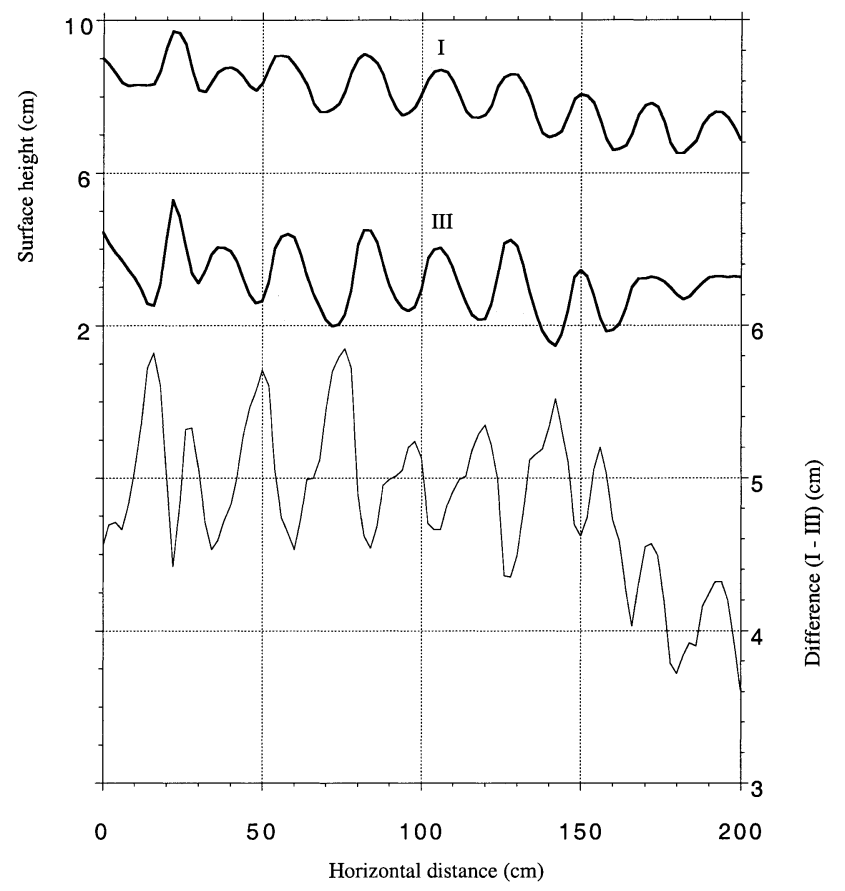

Fig. 6. Measured profiles at R5 on the first (I) and last (III) visits (2-4 February 1998), as well as the difference between the two. This difference reflects the ablation rate during 1 month, which is clearly highest in the troughs.

the two. Evidently, the troughs deepen much more than the crests, while the general waveform remains the same. The maximum difference between trough and crest ablation at R5 is $>1 \mathrm{~cm}$. Obviously, the stake-ablation method, which usually records only the ablation of the crests (when using a horizontal rod to define the surface), will generally underestimate spatially averaged ablation. This was quantified by comparing the average ablation measured at the two stakes by the conventional method (the stake-ablation method), at each site, with the average ablation detectable in the profile measurements (Table 3). The results show that the stake-ablation method generally underestimates true ablation by $6-15 \%$, except at $\mathrm{R} 1$ where it overestimates true ablation (ablation measured at stakes near the weather station at S1 was lower, and would have led to an underestimate of the ablation at R1, as will be discussed below; this illustrates the uncertainties involved in the ablation measured using stakes). Total ablation ranges from 4 to $6 \mathrm{~cm}$

Table 3. Ablation rates (A) between each of the three visits ( cmice $^{-1}$ ) and the total ablation (cmice) over the entire period, as determined from the height difference between the successive profiles

\begin{tabular}{|c|c|c|c|c|c|}
\hline Site & $\begin{array}{c}A \\
(I-I I)\end{array}$ & $\begin{array}{c}A \\
(I I-I I I)\end{array}$ & $\begin{array}{c}A \\
(I-I I I)\end{array}$ & $\begin{array}{c}\text { Total } \\
(I-I I I)\end{array}$ & $\begin{array}{c}\text { Crest } \\
(I-I I I)\end{array}$ \\
\hline $\mathrm{Rl}$ & $0.224(+17)$ & $0.100(-6)$ & $0.162(+6)$ & 5.62 & -3.2 \\
\hline $\mathrm{R} 2$ & $0.118(-17)$ & $0.101(+13)$ & $0.110(-7)$ & 4.35 & -5.1 \\
\hline $\mathrm{R} 3$ & - & - & - & - & - \\
\hline $\mathrm{R} 4$ & - & - & $0.144(-6)$ & 5.20 & -3.3 \\
\hline R5 & - & - & $0.121(-15)$ & 4.95 & -7.5 \\
\hline
\end{tabular}

Notes: Values in brackets represent the error (\%) if stake readings had been used (a negative value indicates that stake ablation is lower than profile ablation). The last column gives the error $(\%)$ if only the profile crests were used to determine the ablation. 
over the entire period, the highest values being found in the low eastern part of the BIA near Rl and R4. This spatial pattern in the ablation rate over the Scharffenbergbotnen BIA agrees with the stake-ablation method observations carried out by Näslund (1992) over a period of 4 years, and can be attributed to warmer and drier conditions, and hence higher sublimation rates, at R1 compared to R2. Bintanja (2000c) used meteorological data from S1 and S2 to calculate the surface energy balance, and arrived at mean sublimation

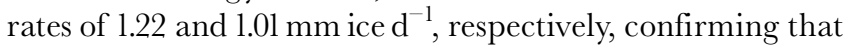
sublimation at $\mathrm{S}$ is higher than at $\mathrm{S} 2$. A consistent feature at all sites is that sublimation is highest in daytime.

From the average profile ablation and the mean crest ablation rates, one can estimate the error in measured ablation if a (hypothetical) stake had been used to determine the ablation rates of the profile crests only (the stake-ablation method). The last column of Table 3 shows this error. The results are quite conclusive, with crest ablation rates consistently smaller than average ablation at all sites. This is roughly in concert with the comparison using real stakes, except at R1. This confirms, as explained before, that the short-term ablation of blue ice as measured via the stakeablation method underestimates true ablation in summer.

The profile measurements at $\mathrm{R} 1$ can be compared with the traditional ablation readings (stake-ablation method) taken near meteorological station S1 and with ablation measurements obtained using an acoustic height sensor (Bintanja and others, 1998). The latter is fixed on a frame drilled into the ice and measures the vertical distance from the sensor to the surface (about $80 \mathrm{~cm}$ initially) by transmitting and receiving sound pulses. The sensor samples a circular area with a diameter of about $40 \mathrm{~cm}$, which thus includes two wavelengths. Like the stake-ablation method, this technique probes only the highest surface elements, as these cause the first return pulse detected by the sensor. Figure 7 shows that the sensor readings yield higher ablation rates than the profile and stake-ablation methods. For 18 January -4 February, the ice-ablation values recorded at $\mathrm{S} 1$ and $\mathrm{R} 1$ by the acoustic sensor, the average ablation values obtained by the traditional stake-ablation method, and the ablation determined by the profile method were 1.67, 1.69 and $1.78 \mathrm{~cm}$, respectively. The two conventional ablation measurement techniques agree well, but underestimate true blue-ice ablation by about $5-6 \%$ at this location. This concurs with the results at the other sites (Table 3).

The general ripple evolution during summer raises an intriguing question relating to ice-ripple evolution during winter. Clearly, the increase in wave height as observed in summer cannot continue throughout the year. Hence, the wave height of the ripples must somehow reduce during the winter, when sublimation rates are low but generally nonnegligible (Bintanja and Reijmer, 2001). Perhaps semipermanent snow coverage in the ripple troughs occurs more regularly during winter, which would leave only the crests exposed to sublimation. However, it is impossible to answer this question with any certainty at present since we have summer observations only. An account of this phenomenon awaits future year-round ripple observations.

\subsection{Ripple orientation}

The orientation of the ripples was determined simply by compass. This was done several times and by two persons independently to enhance accuracy. Generally, winds inside

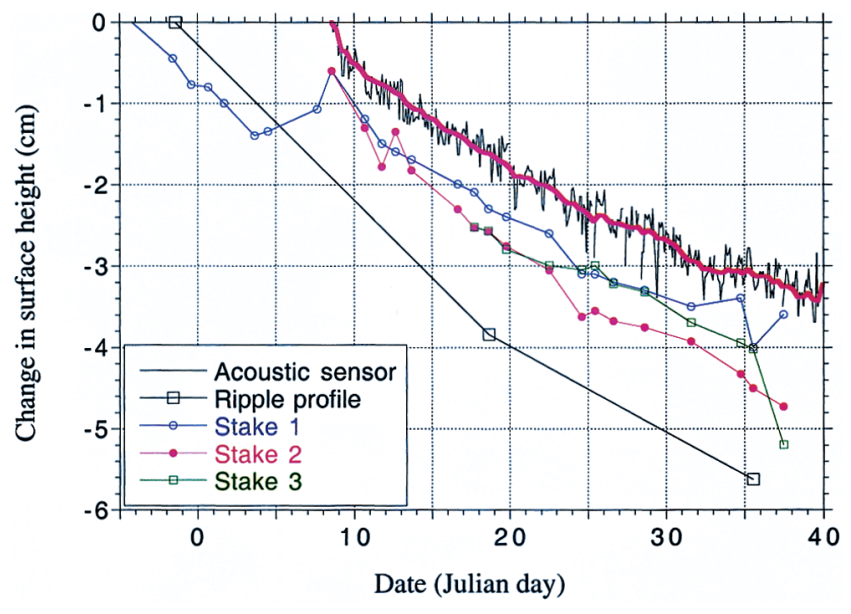

Fig. 7. Ablation near R1 during fanuary 1998 as measured using (1) the acoustic height sensor of the AWS, which samples every $2 \mathrm{~h}$ (the bold red line indicates the 11-point running mean), (2) the ripple profiles at the three visits, and (3) the three stakes close to the weather station (stake observations were carried out every 2-3 days). Notice that these stakes apparently underestimate ablation, whereas the ablation measured at the stakes used to measure ripple profiles exhibited an overestimation.

the Scharffenbergbotnen valley can be subdivided into two categories (Bintanja, 2000a): (1) weak westerly winds with relatively small sublimation rates, and (2) stronger easterlies with high sublimation rates. Additionally, the irregular orography of the surrounding mountains affects the wind direction throughout the valley, resulting in different mean wind directions at S1 and S2. A period-average value was calculated, as well as a mean wind direction for the highest $10 \%$ of $30 \mathrm{~min}$ mean wind-speed values. The sites close to the northeasterly valley wall (R1, R4 and R5) experience southeasterly winds, whereas R2 and R3 located closer to the centre line of the valley experience easterly winds. Table 4 shows the results. Evidently, the ripple crests and troughs are oriented perpendicular to the direction of the strongest winds within the accuracy of the measurements. At Rl and $\mathrm{R} 4$, the ripple pattern is oriented in a more southeasterly direction, in good agreement with the wind directions measured at S1. At R2, the orientation of the ripples corresponds well with the wind direction of the strongest (easterly) winds, while the mean wind direction is more northeasterly. Evidently, little sublimation and ripple formation takes place during the relatively weak westerly

Table 4. Orientation of the ripple direction (taken perpendicular to crest alignment) determined at two visits (I and III), the mean wind direction (MWD) at $S 1$ (close to R1 and R4) and $S 2$ (close to R2) and the wind direction during strong winds (SWD). See text for further explanation

\begin{tabular}{ccccc}
\hline Site & $I$ & III & MWD & SWD \\
\hline R1 & $124 \pm 4$ & $127 \pm 4$ & 144 & $130 \pm 12$ \\
R2 & $103 \pm 2$ & $102 \pm 5$ & 66 & $101 \pm 9$ \\
R3 & $100 \pm 2$ & $108 \pm 7$ & - & - \\
R4 & $122 \pm 2$ & $125 \pm 2$ & 144 & $130 \pm 12$ \\
R5 & $130 \pm 4$ & $127 \pm 2$ & - & -
\end{tabular}

Notes: Directions are in degrees with respect to true north (e.g. east is $\left.90^{\circ}\right)$. The error in an individual ripple direction observation is estimated at $5^{\circ}$. The error denotes the standard deviation of the mean value. 


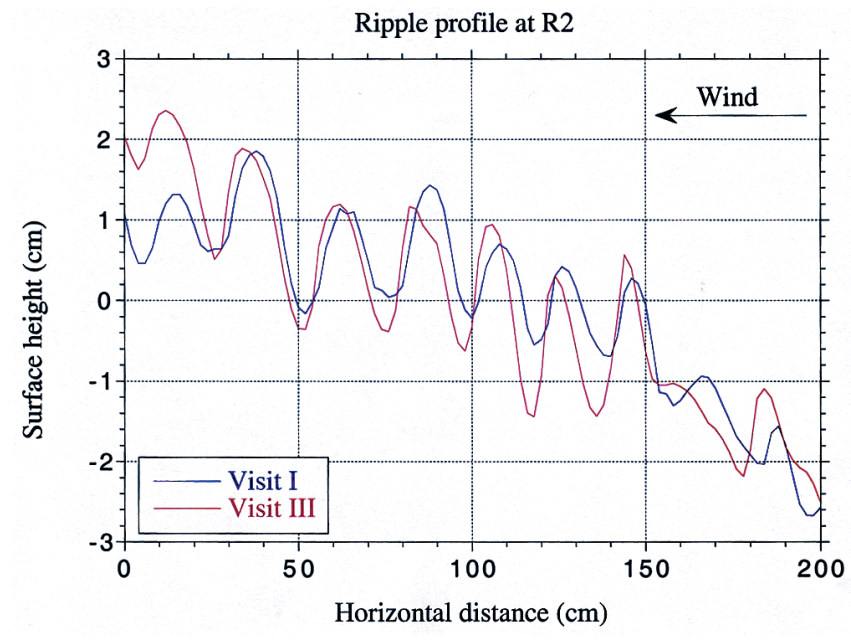

Fig. 8. Ripple profiles at $R 2$ (with the mean value subtracted) measured on the first and last visits. The phase difference suggests a downwind migration of the ripples.

winds, which contribute to the mean values at this site. At $\mathrm{R} 1$ and $\mathrm{R} 4$, the direction of the ripples also corresponds better with the direction of the strongest winds than with that of the mean winds. The overall good agreement between ripple orientation and the direction of the strongest surface winds indicates that the existence of the ripples is definitely linked to the interaction between the ice surface and atmospheric near-surface flow. This also provides an indirect method to estimate the spatial pattern of the direction of the strongest winds: measuring BIA ripple orientation.

\subsection{Ripple migration}

An interesting question is whether blue-ice ripples migrate laterally. If somehow the phase of the spatial pattern in ablation rate is different from that of the actual wave, the ripple pattern will undergo a lateral movement (due to differential ablation). In other words, if the ablation on the lee side of the crest is larger than that on the upwind side then the wave will move in a downwind direction. Consider Figure 8, which shows the first (I) and last (III) measured profile at R2, minus the mean value. The entire profile obviously appears to have moved in the downwind direction, as becomes particularly clear in the comparison of individual crest and trough positions. But is this apparent migration significant? (Note that ice flow has no effect on these results, as both stakes move with the ice; the measurements were made within a coordinate system which would move along with the ice flow, and spatial gradients in ice velocity are extremely small on this BIA.)

In order to quantify any horizontal movement of the ripples, the individual profiles (with the mean value subtracted, and scaled with their variance) measured at different visits were correlated with each other. In each comparison of two individual profiles, one of the two was shifted $1-5$ measuring points $(2-10 \mathrm{~cm})$ to either side. This procedure was followed for each site, except for site 3 . If the maximum correlation occurs at zero shift, then no significant lateral migration has occurred. If, on the other hand, maximum correlation is obtained at other shift values, then the entire profile has apparently moved. Figure 9 shows the correlation coefficients for the various sites and periods. $\mathrm{R} 2$ is the only site at which the ripples appear to
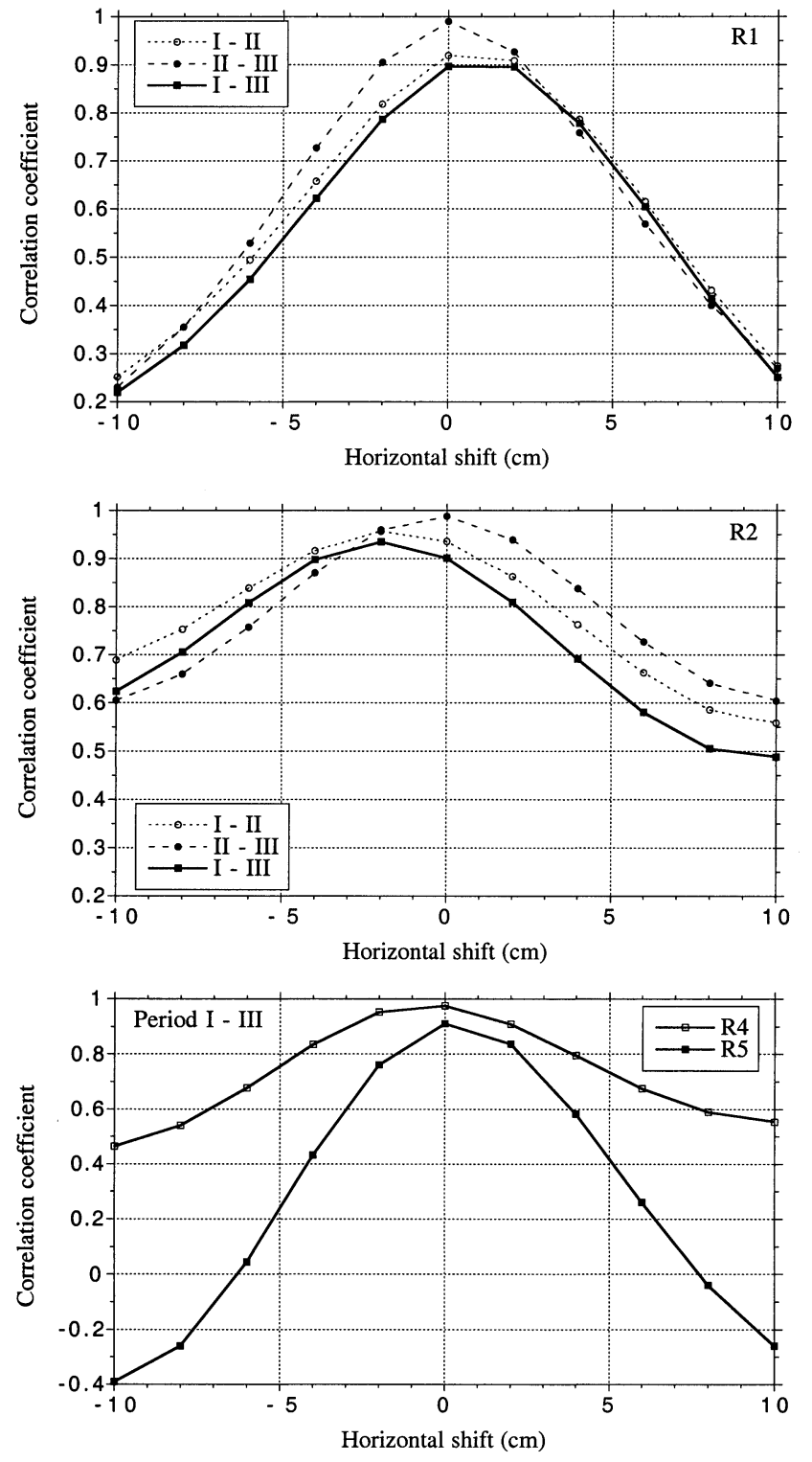

Fig. 9. Correlation coefficients $(R)$ between two profiles measured at different times for horizontal shifts of the second profile relative to the first profile. $R$ is defined as

$R=\left[\sum_{k=1}^{N}\left(x_{k-i}-\bar{x}\right)\left(y_{k}-\bar{y}\right)\right]\left[\sum_{k=1}^{N}\left(x_{k-i}-\bar{x}\right)^{2} \sum_{k=1}^{N}\left(y_{k}-\bar{y}\right)^{2}\right]^{-\frac{1}{2}}$,

where $x$ and $y$ represent the height of the two profiles, $i$ is the horizontal shift and $\mathcal{N}$ is the total number of points (101). In each case, one of the profiles was shifted 1-5 points (2-10 cm) to either side, and the correlation between the two profiles was determined. At R2, the maximum value in $R$ for the first and last profiles occurs at $-2 \mathrm{~cm}$, which indicates a downwind migration of $2 \mathrm{~cm}$ during the 35 day interval.

have migrated over a measurable distance. The shift at R2 amounts to about $2 \mathrm{~cm}$ in the downwind direction, as could be anticipated from the profiles shown in Figure 8. Most of this shift occurred during the first half of January. The other sites show no significant movement, although there is some indication of upwind migration, of $<2 \mathrm{~cm}$, at site 1 . Had we sampled at $1 \mathrm{~cm}$ intervals, we probably would have found a maximum correlation at $1 \mathrm{~cm}$, in the windward direction.

Of course, the lateral displacement of ripples is significant only if the mean vertical difference in successive data points is larger than the accuracy of the individual measurements. The value of the former is $0.2-0.3 \mathrm{~cm}$, which is larger than the 
accuracy of the measurements (i.e. $0.05 \mathrm{~cm}$ ). The downwind migration of the rippled structure at $\mathrm{R} 2$ at a rate of about $2 \mathrm{~cm} \mathrm{month}^{-1}$ is therefore indeed significant. At present, it is unclear why the ripples at S2 did migrate, whereas the ripples at the other sites did not exhibit a (significant) lateral movement. Obviously, taking a larger temporal interval between measurements or extending the sampling might help answer this question. In any case, our observations show that the possibility of blue-ice ripple migration is real. Any future attempts to measure the characteristics of BIA ripples should involve the observation of wave migration.

\section{A SIMPLE MODEL OF ICE-RIPPLE GENERATION}

The observations presented in the previous section clearly indicate that the surface airflow is a key factor in the formation of ripples. In this section we investigate whether the observed blue-ice ripples can be caused by a free instability of the coupled ice-airflow system. To test this, we formulate a very simple model and apply a linear stability analysis. Such an analysis provides insight into the typical wavelength and migration speed of those blue-ice ripples that are most likely to exist. Moreover, it allows an interpretation of the near-surface airflow over the modelled surface patterns and thereby helps to identify the fundamental mechanisms that cause blue-ice ripples to form.

\subsection{Description of the model}

We assume cross-ripple uniformity and take the following, simplest possible, model able to describe the flow in the vertical plane $(x, z)$ just above the surface:

$$
\begin{gathered}
u \frac{\partial u}{\partial x}+w \frac{\partial u}{\partial z}=K \frac{\partial^{2} u}{\partial z^{2}} \\
\frac{\partial u}{\partial x}+\frac{\partial w}{\partial z}=0,
\end{gathered}
$$

where $x$ is the horizontal coordinate perpendicular to the ripple crests (and parallel to the mean wind direction), $z$ is the vertical coordinate, and $u$ and $w$ are the horizontal and vertical components of the the wind. Downwind and upward directions are taken positive. Equations (2) and (3) govern the conservation of horizontal momentum and continuity, respectively. The turbulent viscosity is given by $K\left(=1 \times 10^{-3} \mathrm{~m}^{2} \mathrm{~s}^{-1}\right)$, which is assumed independent of height. The change in surface height $(h)$ is described by:

$$
\frac{\partial h}{\partial t}=-\left.b u\right|_{z=h}+c
$$

where the first term on the righthand-side (typically, $b \cong$ $1.5 \times 10^{-8}$ at $\mathrm{S} 1$ and $\mathrm{S} 2$ ) represents the sublimation rate, which is taken proportional to the velocity just above the viscous sublayer, and the second term $\left(c \cong 3 \times 10^{-9} \mathrm{~m} \mathrm{~s}^{-1}\right)$ equals the continuous overall upward movement of the ice surface. In steady state, $c$ equals the ablation rate which at $\mathrm{S} 1$ and $\mathrm{S} 2$ is about $10 \mathrm{~cm}$ w.e. $\mathrm{a}^{-1}$.

The ice surface evolves slowly (i.e. $\partial h / \partial t \ll w$ ) so that just above the ice surface $(z=h)$ :

$$
\left.w\right|_{z=h}=\left.u\right|_{z=h} \frac{\partial h}{\partial x} \quad \text { and }\left.\quad K \frac{\partial u}{\partial z}\right|_{z=h}=\left.s u\right|_{z=h},
$$

where $s$ is a parameter that can be interpreted in terms of a friction velocity. It is included to ensure that the shear stress at the surface attains realistic values. The first condition ensures that the flow follows the evolving surface. Far away from the ice surface $(z \rightarrow \infty)$ we assume that the shear stress $(\tau)$ is constant with height:

$$
\left.\frac{\partial u}{\partial z}\right|_{z \rightarrow \infty}=\frac{\tau}{\rho K} \equiv B
$$

where $\rho$ is the air density. The simple ice-surface-airflow system is now complete: we can solve the set of prognostic variables $\Psi=\{u, w, h\}$ by using Equations (2), (3) and (4), supplemented with the boundary conditions (5) and (6). It should be noted that this is probably the simplest possible model to study the formation of ice ripples in a physically realistic manner.

\subsection{Basic state: a flat ice surface}

The stability analysis involves a perturbation of a basic state (subcript 0). Here, we take a basic solution which is horizontally uniform (i.e. $\partial \Psi_{0} / \partial x=0$ ). Physically, this refers to a flat horizontal ice surface $\left(h_{0}=0\right)$ without vertical motion $\left(w_{0}=0\right)$. This leads to a horizontal flow that increases linearly with height:

$$
u_{0}=A+B z,
$$

where

$$
\sqrt{K B}=\frac{c}{b} \quad \text { and } \quad A=\frac{\tau}{\rho s}=\frac{B K}{s} .
$$

We should note that extension of a linear velocity profile beyond the viscous sublayer is not very realistic, even though we do believe that the precise form of the velocity profile is not vital. The linear profile was chosen because it facilitates an easy solution of the model.

\subsection{Perturbations of the basic state}

We perturb the basic state by allowing for arbitrary perturbations. Mathematically this means substitution of $\Psi=\Psi_{0}+\Psi_{1}$ in which $\left\|\Psi_{1}\right\| \ll\left\|\Psi_{0}\right\|$. Using the structure of the starting equations, the basic state, Equations (2-8) and a Taylor expansion at the boundary conditions (see, e.g., Hulscher, 1996), this leads to a problem for $\Psi_{1}$ in which the air circulation is related to the existence of surface ripples of a specified wavelength.

Primarily, we investigate sinusoidal ice-forms having wavenumber $k$, so that the perturbed problem can be analyzed after Fourier transformation, by defining $\boldsymbol{\Psi}=(\mathbf{u}, \mathbf{w}, \mathbf{h})$ as:

$$
\Psi_{1}(x)=\int \boldsymbol{\Psi}(k) \mathrm{e}^{-i k x} \mathrm{~d} x+\mathrm{cc},
$$

where cc is the complex conjugate.

\subsection{The growth rate of surface ripples}

The perturbed problem splits into two parts which can be solved one after the other. The first part involves the reaction of the flow to a rippled ice surface of wavelength $2 \pi / k$ and initial amplitude $\mathbf{h}$. This problem is solved here using a combination of approximation and numerical techniques (for details, see analogous sand-wave models (e.g. Hulscher, 1996; Komarova and Hulscher, 2000)). The second part of the perturbation problem deals with the amplification or damping of the initial ice-ripple perturbation $\mathbf{h}$, given by

$$
\frac{\partial \mathbf{h}}{\partial t}=\omega \mathbf{h},
$$

where $\omega=\operatorname{Im}(\omega)+\operatorname{Re}(\omega)$ is the complex growth rate that is composed of an imaginary and a real part, respectively. 


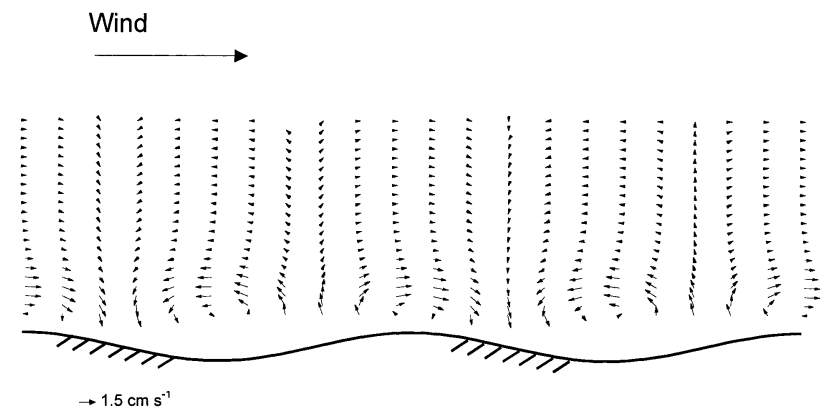

Fig. 10. Pattern of the perturbed flow over blue-ice ripples with a wavelength of $20 \mathrm{~cm}$ as calculated by the model. The horizontal and vertical dimensions of the plot are 0.4 and $0.1 \mathrm{~m}$, respectively. The background flow is from left to right, which implies that the airflow (and sublimation rate) over ripple crests (troughs) is diminished (enhanced). This favours ripple growth.

Solving this equation and making use of the Fourier transform (Equation (9)), we arrive at

$$
h(x, t)=\frac{\mathbf{h}}{2} \mathrm{e}^{\operatorname{Re}(\omega) t} \cos (k x-\operatorname{Im}(\omega) t) .
$$

This means that the ripple migration speed equals $\operatorname{Im}(\omega) / k$. The real part of the growth rate $\operatorname{Re}(\omega)$ governs the amplification $(\operatorname{Re}(\omega)>0)$ or damping $(\operatorname{Re}(\omega)<0)$ of the ripples.

\subsection{Results}

We allow for a non-zero airflow close to the surface and take $A=0.4 \mathrm{~m} \mathrm{~s}^{-1}$ and $B=35 \mathrm{~s}^{-1}$, which provides a background flow that, in the region of interest, agrees well with the logarithmically extrapolated wind speeds that were measured at $\mathrm{S} 1$ and $\mathrm{S} 2$. Figure 10 shows the perturbation flow field over the ice surface for the case $k=31.4 \mathrm{~m}^{-1}$ (i.e. a wavelength of $20 \mathrm{~cm}$ ), which is a typical flow pattern over ice ripples that grow in time. As can be seen, crests experience weaker flow than would be the case in the absence of ripples, and troughs stronger flow. As a result, sublimation decreases at the crests and increases in the troughs, and ripple growth occurs. This spatial pattern in sublimation allows the amplitude of these ripples to grow in time. Incidentally, the model only specifies whether ripples with certain wavelengths can initially grow. Once ripples are formed, other, probably nonlinear, mechanisms will counteract ripple growth so that a certain optimum wave height is achieved. Modelling these effects is far beyond the possibilities of this model, so we are unable to predict ripple wave heights.

For shorter wavelengths, the situation is reversed, and the sublimation over crests is larger than in the troughs. Hence, according to the model, ripples with shorter wavelengths cannot grow. The transition from negative horizontal perturbation flow (decrease of the background flow) over the ice crests to a positive horizontal perturbation flow (increase of the background flow) over the ice crests is found at $k=40 \mathrm{~m}^{-1}$ (i.e. a wavelength of $16 \mathrm{~cm}$ ). This is reflected in the change of sign of the real part of the growth rate (Fig. 11), with positive values at higher wavelengths indicating growth of the amplitude of the ripples. Hence, the model predicts that wavelengths shorter than $16 \mathrm{~cm}$ will never form under the specific atmospheric conditions imposed here. Thus, spatial variations in shear stress and sublimation rate caused by a specific surface pattern determine whether ripples of a certain

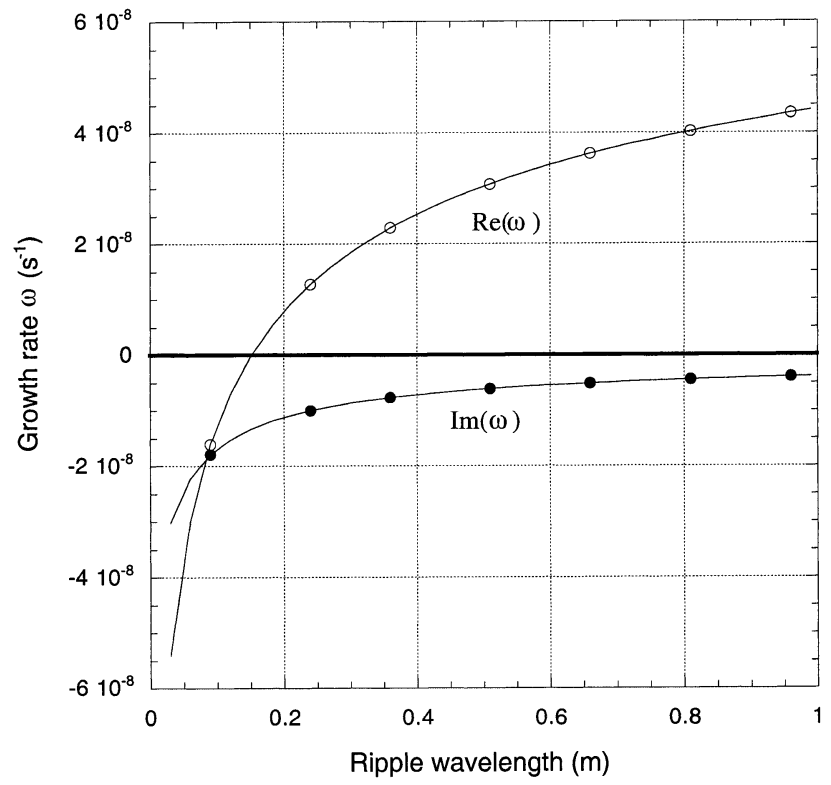

Fig. 11. Real and imaginary parts of the growth rate (see Equations (10) and (11)) as a function of ripple wavelength. Positive values indicate ripple growth and downwind migration of ripples, respectively.

wavelength can grow. Such spatial differences exist because of the presence of circulation cells in the perturbation flow field in the boundary layer above the rippled ice surface.

The imaginary part of the growth rate (Fig. 11) is always negative. This means that, according to this model, the ripples always migrate in the upwind direction. For a ripple having a wavelength of $20 \mathrm{~cm}$ we find $\operatorname{Im}(\omega) \approx-2 \times 10^{-8} \mathrm{~s}^{-1}$, which is equivalent to a migration rate of $1.5 \mathrm{~mm} \mathrm{month}^{-1}$ in a direction opposite to the background flow. This small migration speed might explain why the ripples at most sites did not exhibit a significant migration speed over the 1 month measuring period.

The results of this simple model agree reasonably well with the observed ripple characteristics presented in section 3. Ripples of the observed wavelength of $20 \mathrm{~cm}$ grow in time, and wavelengths shorter than $16 \mathrm{~cm}$ are suppressed. Apparently, this simple model does not contain mechanisms by which longer wavelengths are suppressed, so that a maximum growth rate (at the wavelength that will actually form) could not be established. The small migration rate for ripples with the observed wavelength seems to be of the correct order of magnitude (too small to be detected within a 1 month period), but the direction of movement is contrary to the observations at S2. A future modelling attempt will include an explicit moisture equation so that the sublimation rate can be calculated more accurately.

\section{GONGLUDING REMARKS}

In this paper, we have presented results of detailed observations of surface ripples found at a blue-ice area near Svea. We have profiled $2 \mathrm{~m}$ stretches of surface topography at five sites during austral summer 1997/98. These measurements were repeated once or twice within 1 month to reveal possible temporal variations, and were complemented by more conventional ablation measurements such as stakes and an acoustic height sensor. The origin and characteristics of the regular ripple forms are probably related to the interaction between the free ice surface and the overlying atmosphere 
through spatial variations in sublimation. The formation of blue-ice ripples is probably analogous to the scallops and ripples that have been observed to form in many types of flows over evaporative or soluble walls (Thomas, 1979). The observations presented here are supplemented by the results of a simple model. The list below gives a summary of the findings presented in this paper.

The wavelength of the ripples was 20-24 cm, and exhibits little spatial variation. Most of the surface of the blue ice is rippled.

The wave height (crest-trough) was $1-2 \mathrm{~cm}$. It increased with time during the summer experiment, with increases of up to $60 \%$ within 1 month. Crests experienced significantly less sublimation than troughs, i.e. the surface ripples become deeper in summer.

Ablation as measured by the traditional stake method and by an acoustic height sensor generally underestimates true ablation by $6-15 \%$ because of the deepening of the ripples during summer. It can be assumed that annual average wave height does not vary over the years. This implies that wave-height increase during the summer is compensated during the winter. However, the mechanisms by which this could be achieved are uncertain.

The orientation of the crests is perpendicular to that of the direction of the strongest winds, which are thought to contribute most to ripple formation through strong sublimation. Hence, wind-induced sublimation is the process that governs ripple formation. Conversely, this allows one to estimate the predominant wind direction over blue-ice areas from the orientation of the surface ripples.

One of the sites exhibited a migration of the ripple pattern of $2 \mathrm{~cm}$ in the downwind direction, whereas other sites showed no significant ripple movement. Preferably, measurements should be done throughout the year to be able to determine the wave speed of the blue-ice ripples with any accuracy. Ripple migration may be attributable to differences in sublimation rates between the upwind and downwind sides of the crests and hence differential ablation.

A simple model was used to quantify the interaction between the rippled ice surface, the atmospheric circulation aloft and the sublimation rate. The model results confirm that blue-ice ripples with the observed wavelength of $20 \mathrm{~cm}$ are able to grow, and that ripples with wavelengths smaller than $16 \mathrm{~cm}$ are suppressed. The modelled migration speed of $1.5 \mathrm{~mm}$ month $^{-1}$ agrees with observed migration rates (at four sites) to the extent that it is too small to be detected. The modelled direction of migration is upwind, which is in contrast to what has been observed at one site. Measurements over a longer period are required to validate the modelled migration rate and direction. It is expected that a more realistic treatment of the mechanisms that govern blue-ice ripple formation will further improve these model predictions.

\section{ACKNOWLEDGEMENTS}

We are grateful to W. Boot and H. Snellen, who did most of the work in designing the specific measuring devices. M. van den Broeke, R. van de Wal and two reviewers made useful comments on earlier versions of the paper. Financial support was provided by the Netherlands Antarctic Research Programme (ALW), which is part of the Netherlands Organization of Scientific Research (NWO).

\section{REFERENGES}

Bintanja, R. 1999. On the glaciological, meteorological and climatological significance of Antarctic blue-ice areas. Rev. Geophys., 37 (3), 337-359.

Bintanja, R. 2000a. Mesoscale meteorological conditions in Dronning Maud Land, Antarctica, during summer: a qualitative analysis of forcing mechanisms. F. Appl. Meteorol., 39(12), 2348-2370.

Bintanja, R. 2000b. Mesoscale meteorological conditions in Dronning Maud Land, Antarctica, during summer: the momentum budget of the boundary layer. Antarct. Sci., 12(2), 229-242.

Bintanja, R. 2000c. The surface heat budget of Antarctic snow and blue ice: interpretation of temporal and spatial variability. 7. Geophys. Res., 105 (D19), 24,387-24,407.

Bintanja, R. and C.H. Reijmer. 2001. Meteorological conditions over Antarctic blue-ice areas and their influence on the local surface mass balance. 7. Glaciol., 47(156), 37-50.

Bintanja, R. and M. R. van den Broeke. 1995. The surface energy balance of Antarctic snow and blue ice. F. Appl. Meteorol., 34(4), 902-926.

Bintanja, R., S. Jonsson and W. H. Knap. 1997. The annual cycle of the surface energy balance of Antarctic blue ice. f. Geophys. Res., 102(D2), 1867-1881.

Bintanja, R., C. H. Reijmer, H. Snellen and M. P. A. Thomassen. 1998. Meteorological and glaciological investigations on a blue-ice area in the Heimefrontfjella, Dronning Maud Land, Antarctica: the follow-up of the 92-93 experiment. Utrecht, Utrecht University. Institute for Marine and Atmospheric Research. (199798 field report.)

Blondeaux, P. 1990. Sand ripples under sea waves. Part 1. Ripple formation. f. Fluid Mech., 218, 1-17.

Cassidy, W. A., R. Harvey, J. Schutt, G. DeLisle and K. Yanai. 1992. The meteorite collection sites of Antarctica. Meteoritics, 27(5), 490-525.

Grootes, P. M. $1990 .{ }^{18} \mathrm{O}$ results from blue-ice areas: "old" ice at the surface? In Cassidy, W. A. and I. M. Whillans, eds. Workshop on Antarctic Meteorite Stranding Surfaces held at University of Pittsburgh, Fuly 13-15, 1988. Houston, TX, Lunar and Planetary Institute, 67-69. (LPI Technical Report.)

Hulscher, S. J. M. H. 1996. Tidal-induced large-scale regular bed form patterns in a three-dimensional shallow water model. 7. Geophys. Res., 101 (C9), 20,727-20,744.

Komarova, N. L. and S. J. M. H. Hulscher. 2000. Linear instability mechanisms for sand wave formation. F. Fluid Mech., 413, 219-246.

Lliboutry, L. 1954. The origin of penitents. F. Glaciol., 2(15), 331-338.

Mellor, M. and C. Swithinbank. 1989. Airfields on Antarctic glacier ice. CRREL Rep. 89-21.

Näslund, J.-O. 1992. Blue-ice investigations in the Scharffenbergbotnen basin. In Melander, O. and M. L. Carlsson, eds. Swedish Antarctic Research Programme 1991/92. Stockholm, Swedish Polar Research Secretariat, 48-53. (Cruise Report.)

Rhodes, J. J., R. L. Armstrong and S. G. Warren. 1987. Mode of formation of "ablation hollows" controlled by dirt content of snow. F. Glaciol., 33 (114), 135-139.

Schytt, V. 1961. Glaciology II(E). Blue ice-fields, moraine features and glacier fluctuations. Norwegian-British-Swedish Antarctic Expedition, 1949-52. Sci. Results IV, 181-204.

Thomas, R. M. 1979. Size of scallops and ripples formed by flowing water. Nature, 277, 281-283.

Van den Broeke, M. R. and R. Bintanja. 1995. The interaction of katabatic winds and the formation of blue-ice areas in East Antarctica. F. Glaciol., 41 (138), 395-407.

Van Roijen, J. J., K. van der Borg, A. F. M. de Jong and J. Oerlemans. 1995. Ages and ablation and accumulation rates from ${ }^{14} \mathrm{C}$ measurements on Antarctic ice. Ann. Glaciol., 21, 139-143.

Weller, G. E. 1968. The heat budget and heat transfer processes in Antarctic plateau ice and sea ice. ANARE Sci. Rep., Ser. A(4) Glaciology 102.

Winther, J.-G., H. Elvehøy, C. E. Bøggild, K. Sand and G. Liston. 1996. Melting, runoff and the formation of frozen lakes in a mixed snow and blue-ice field in Dronning Maud Land, Antarctica. f. Glaciol., 42(141), 271-278. 